THE PRINCETON ANTHOLOGY OF WRITING 



\section{The Princeton Anthology of}

Writing Favorite Pieces by the Ferris/McGraw

Writers at Princeton University

\section{John McPhee AND Carol Rigolot}

EDITORS 
Copyright (C) 2001 by Princeton University Press

Published by Princeton University Press, 41 William Street,

Princeton, New Jersey 08540

In the United Kingdom: Princeton University Press, 3 Market Place,

Woodstock, Oxfordshire OX20 1SY

All Rights Reserved

Library of Congress Catalog Card Number 2001089961

ISBN 0-691-08680-X (cloth)

ISBN 0-691-08681-8 (alk. paper)

This book has been composed in Berkeley

Designed by Jan Lilly

Composed by Gretchen Oberfranc

Printed on acid-free paper: $\infty$

www.pup.princeton edu

Printed in the United States of America

$\begin{array}{llllllllll}10 & 9 & 8 & 7 & 6 & 5 & 4 & 3 & 2 & 1\end{array}$

$\begin{array}{llllllllll}\text { i0 } & 9 & 8 & 7 & 6 & 5 & 4 & 3 & 2 & 1\end{array}$

(Pbk.) 